\title{
SAFYRE. UN NUEVO CONCEPTO DE CABESTRILLO AJUSTABLE MÍNIMAMENTE INVASIVO PARA LA INCONTINENCIA URINARIA POR ESTRÉS FEMENINA
}

\author{
P.C.R. PALMA*, C.L.Z. RICCETTO*, M. DAMBROS*, M. THIEL*, R. DE FRAGA*, \\ J.T.N. TAMANINI*, V. HERRMANN*, N.R. NETTO JR*, O. GROSSI**, M. ZANGONE**, \\ M. PALADINI**, H. RETTO***, J. COLAÇO***, D. CASTRO DIAZ****
}

*División de Urología, Universidade Estadual de Campinas, UNICAMP. **Clínica Universitaria Reina Fabiola, Universidad Católica de Córdoba, Argentina. ***Servicio de Ginecología, Hospital Garcia de Orta, Almada, Portugal. ${ }^{* * * * H o s p i t a l ~ U n i v e r s i t a r i o ~ d e ~ l a s ~ I s l a s ~ C a n a r i a s, ~ T e n e r i f e . ~ E s p a n ̃ a . ~}$

Actas Urol Esp. 28 (10): 749-755, 2004

\section{RESUMEN}

SAFYRE. UN NUEVO CONCEPTO DE CABESTRILLO AJUSTABLE MÍNIMAMENTE INVASIVO PARA LA INCONTINENCIA URINARIA POR ESTRÉS FEMENINA

INTRODUCCIÓN: SAFYRE es un nuevo cabestrillo (dispositivo de suspensión) reajustable y mínimamente invasivo para el tratamiento de la incontinencia urinaria por estrés (IUE). En los últimos años se ha intentado restaurar el lecho suburetral normal utilizando una técnica anatómica. Los autores comunican su experiencia con este dispositivo, que además de poseer la eficacia de los cabestrillos tiene capacidad de reajustación.

MATERIALES y MÉTODOS: Un total de 100 pacientes consecutivas con diagnóstico de IUE desde el punto de vista clínico y urodinámico se sometieron al procedimiento de implantación del cabestrillo SAFYRE. Su rango de edad era de 40 a 71 años, con una edad media de 63 años. Previamente, 75 pacientes (75\%) se habían sometido a procedimientos para la incontinencia que habian fracasado. Antes de la cirugía se realizaron una exploración física clínica, una prueba de estrés, un estudio urodinámico y un estudio sobre el uso de almohadillas. Todas las pacientes presentaban síntomas de IUE y el 30\% también tenían tenesmo leve.

RESULTADOS: El periodo de seguimiento medio fue de 14 meses (12 - 30 meses). El tiempo medio de la operación fue de 25 minutos. Durante el mismo procedimiento se reparó la distopia cuando se consideró necesario. La estancia hospitalaria media fue de 24 horas. En el 3\% de los implantes se produjo perforación de la vejiga. Durante el periodo posoperatorio 26 pacientes presentaron sintomas de tenesmo. Durante ese periodo de seguimiento, el 92\% presentaron continencia urinaria, el 3\% mejoraron y el $5 \%$ no estuvieron satisfechas con el procedimiento.

CONCLUSIÓN: SAFYRE es un procedimiento seguro y rápido que permite el reajuste posoperatorio. Esta técnica puede ser una alternativa atractiva si se demuestra que los buenos resultados obtenidos hasta ahora son de larga duración.

PALABRAS CLAVE: Incontinencia urinaria por estrés. Cabestrillo. Mujeres. Fracaso.

\section{ABSTRACT}

SAFYRE. A NEW CONCEPT FOR ADJUSTABLE MINIMALLY INVASIVE SLING FOR FEMALE URINARY STRESS INCONTINENCE

INTRODUCTION: SAFYRE is a new readjustable and minimally invasive sling for the treatment of stress urinary incontinence (SUI). Attempts to restore the normal suburethral hammock using an anatomical approach have been made in recent years. The authors report their experience with this device, which associates the efficacy of slings with readjustability.

MATERIAL AND METHODS: A total of 100 consecutive patients with clinical and urodynamic diagnosis of SUI underwent SAFYRE sling procedure. The age range was from 40 to 71 , mean age 63 years. Seventy-five patients (75\%) presented previous failed anti-incontinence procedures. Physical clinical examination, stress test, pad use and a urodynamic study were performed before the surgery. All the patients presented symptoms of SUI and $30 \%$ also reported mild urgency.

RESULTS: The average follow up period was 14 months (12 - 30 months). The mean operative time was of 25 minutes. Dystopia repair was performed whenever necessary, during the same procedure. The average hospital stay was 24 hours. In $3 \%$ of the implants, bladder perforation occurred. During the postoperative period, 26 patients developed urgency symptoms. During that follow up period, $92 \%$ were found to be continent, $3 \%$ reported an improvement and $5 \%$ were dissatisfied.

CONCLUSION: SAFYRE is a safe and quick procedure that allows for postoperative readjustment.

This technique may be an attractive alternative if the good result obtained so far proves to be long lasting.

KEYWORDS: Stress urinary incontinence. Sling. Female. Failure. 
$\mathrm{L}$ os cabestrillos pubovaginales llevan usándose desde principios del siglo pasado ${ }^{1}$. Actualmente se considera que esta técnica es la más eficaz para tratar la incontinencia urinaria por estrés (IUE) en pacientes con lesiones del mecanismo uretral intrínseco ${ }^{2}$. Sin embargo, la morbilidad y aspectos de la convalecencia han estimulado la investigación en procedimientos menos invasivos como los cabestrillos mínimamente invasivos y las inyecciones periuretrales.

Las cintas sintéticas tienen una tasa de éxito elevada y fortalecen los tejidos dañados. Un efecto beneficioso adicional de los cabestrillos sintéticos es que con ellos los procedimientos de cirugía mayor son mínimamente invasivos y también reducen el tiempo de la operación, la estancia hospitalaria y las molestias en el posoperatorio ${ }^{3}$.

Desde la comercialización y el éxito de la técnica TVT, se han desarrollado otros productos para el mercado de cabestrillos suburetrales. El cabestrillo SAFYRE, que posee un sistema de autofijación, se ha añadido recientemente a las herramientas existentes. Es un cabestrillo sintético que no produce tensión que se coloca en la parte media de la uretra y es improbable que cause erosiones uretrales. Es un cabestrillo sintético reajustable con un sistema de autofijación que permite reajustar la tensión en el posoperatorio si se producen fuga o retención urinarias ${ }^{4}$.

Según la teoría de la continencia integral, las regiones del tercio medial y distal de la uretra son las más importantes. El procedimiento SAFYRE se basa en esta teoría y en estudios experimentales del mecanismo del cierre de la uretra en las mujeres ${ }^{5}$.

Los cabestrillos se utilizan ahora aún con más frecuencia, y el SAFYRE, que se basa en estos nuevos conceptos, es una alternativa atractiva para el tratamiento quirúrgico de la IUE. Los autores presentan su experiencia con este cabestrillo reajustable, centrándose en la seguridad en el periodo perioperatorio y en la eficacia a medio plazo del procedimiento.

\section{MATERIALES Y MÉTODOS}

\section{Pacientes}

Llevamos a cabo un estudio clínico prospectivo, multicéntrico, no aleatorizado y con un único grupo en pacientes con IUE, que fue aprobado por el comité ético de investigación clínica del hospital.

Desde febrero de 2001 hasta marzo de 2002, a 100 pacientes con diagnóstico de IUE se les implantó el SAFYRE. Las edades de las pacientes oscilaban entre 40 y 71 años (mediana de edad de 63 años).

\section{Diseño del estudio}

A todas las pacientes se les diagnóstico incontinencia mediante el procedimiento habitual, incluida la historia médica, y luego se les realizó una evaluación del impacto usando la versión abreviada del Cuestionario de la Consulta Internacional sobre la Incontinencia $(\mathrm{ICIQ}-\mathrm{SF})^{6}$, un examen ginecológico, una prueba de estrés, un estudio sobre el uso de almohadillas y una investigación urodinámica. La evaluación urodinámica se llevó a cabo con 2 catéteres uretrales (uno $10 \mathrm{~F}$ para el llenado y otro $4 \mathrm{~F}$ para determinar la presión de la vejiga). Se colocó un catéter de balón rectal $4 \mathrm{~F}$ por encima del esfínter anal para determinar la presión abdominal. La prueba comprendió una cistometría acuosa, la evaluación de la presión del punto de fuga de Valsalva (PPFV), que se realizó con un volumen intravesical de $200 \mathrm{ml}$ y las maniobras de Valsalva, y un estudio de presiónflujo.

El examen ginecológico reveló la presencia de cistocele leve en 62 pacientes (62\%); el $70 \%$ de los casos eran de grado I y el resto de grado II. Se diagnosticó rectocele de grado I en 13 pacientes y solamente se repararon los casos de cistocele sintomático de grado II (3 pacientes).

La prueba de estrés fue positiva en todas las pacientes. Los resultados relacionados con las pruebas urodinámicas se muestran en la Tabla 1.

Tabla 1

Resultados relacionados con la presión del punto de fuga de Valsalva preoperatoria

\begin{tabular}{lcc}
\hline $\begin{array}{l}\text { Presión del punto de fuga } \\
\text { de Valsalva preoperatorio }\end{array}$ & $\begin{array}{c}\text { número } \\
\text { de pacientes }\end{array}$ & $\begin{array}{c}\text { porcentaje } \\
\text { (\%) }\end{array}$ \\
\hline $\begin{array}{l}\left.\text { (media de } 71 \mathrm{cmH}_{2} \mathrm{O}\right) \\
\text { (m) } \mathrm{cmH}_{2} \mathrm{O}\end{array}$ & 54 & 43 \\
$91-125 \mathrm{cmH}_{2} \mathrm{O}$ & & \\
$\left(\right.$ media de $\left.99 \mathrm{cmH}_{2} \mathrm{O}\right)$ & 72 & 57 \\
\end{tabular}


Del estudio se excluyó a las pacientes que presentaron contracciones involuntarias del detrusor durante el llenado de la vejiga o un flujo máximo (Qmax) de menos de $15 \mathrm{ml} / \mathrm{s}$ y/o un volumen de orina residual tras la micción de más del $20 \%$ del volumen miccionado, pero se incluyó a las que presentaron síntomas de irritación sin contracciones involuntarias demostradas mediante urodinámica. Aunque la inestabilidad del detrusor demostrada mediante urodinámica no tiene un efecto significativo sobre el resultado quirúrgico, esta decisión se basó en el concepto de la mejora en el posoperatorio del tenesmo sensorial, tal como se ha descrito previamente ${ }^{7}$. De este estudio inicial se excluyó a las pacientes con contracciones involuntarias del detrusor debido a que su pronóstico es menos favorable en lo referente a los síntomas de irritación en el posoperatorio ${ }^{8}$.

La mayoría de las pacientes de este ensayo clínico se habían sometido previamente a al menos un procedimiento para la incontinencia que había fracasado $(75 \%)$. El procedimiento realizado con más frecuencia fue la reparación vaginal anterior (Tabla 2).

El seguimiento se realizó al cabo de 1 mes y cada 6 meses. En estas visitas, se preguntó a las pacientes si habían presentado micción espontánea, fuga urinaria involuntaria, síntomas de irritación de la vejiga y dolor vaginal y suprapúbico, y luego se les hizo una evaluación del impacto, una prueba de estrés y una evaluación del uso de almohadillas.

Los resultados quirúrgicos se clasificaron en 3 categorías según los criterios de Blaivas y Jacobs $^{9}$ : a) curación - ausencia de incontinencia;

\section{Tabla 2}

Procedimientos quirúrgicos previos para la incontinencia urinaria por estrés

\begin{tabular}{lcc}
\hline Técnica & N & (\%) \\
\hline Ninguna & 50 & 39,7 \\
Reparación anterior (plicación de Kelly) & 37 & 29,4 \\
Colposuspensión retropúbica & 12 & 9,5 \\
Cabestrillo pubovaginal & 12 & 9,5 \\
Inyección periuretral & 8 & 6,5 \\
Suspensión con aguja & 7 & 5,5 \\
\hline
\end{tabular}

b) mejoría - frecuencia de episodios de incontinencia de menos de uno cada 2 semanas; c) fracaso - frecuencia de episodios de incontinencia de más de uno a la semana.

\section{Materiales}

El SAFYRE consiste en una malla de polipropileno que actúa como un soporte uretral, sostenido entre dos columnas de autofijación de polímero de polidimetilsiloxano de grado para implante. Estas columnas son la base del sistema de autofijación. Con el fin de minimizar la lesión quirúrgica de las estructuras de soporte naturales del ligamento inferior de la pelvis se utiliza una aguja especial de $3,5 \mathrm{~mm}$ de diámetro que permite el abordaje suprapúbico y transvaginal, dependiendo de la experiencia del cirujano (Fig. 1). Para el abordaje transvaginal, el extremo en forma de gancho se introduce dentro de la boquilla de la aguja versátil, y para el abordaje suprapúbico se hace de la otra manera (la aguja y el cabestrillo los fabrica Promedon, Córdoba, Argentina).

\section{Técnica quirúrgica}

El procedimiento se realizó con la paciente en posición de litotomía bajo anestesia espinal.

Se administraron por vía intravenosa 2 gramos de cefalosporina de primera generación en el momento de inducción de la anestesia y luego 1 gramo 6,12 y 18 horas después del procedimiento.

Se hacen dos incisiones transversales de 0,5 $\mathrm{cm}$ cerca de la cara superior del hueso púbico separadas entre ellas por $5 \mathrm{~cm}$. Se realiza una

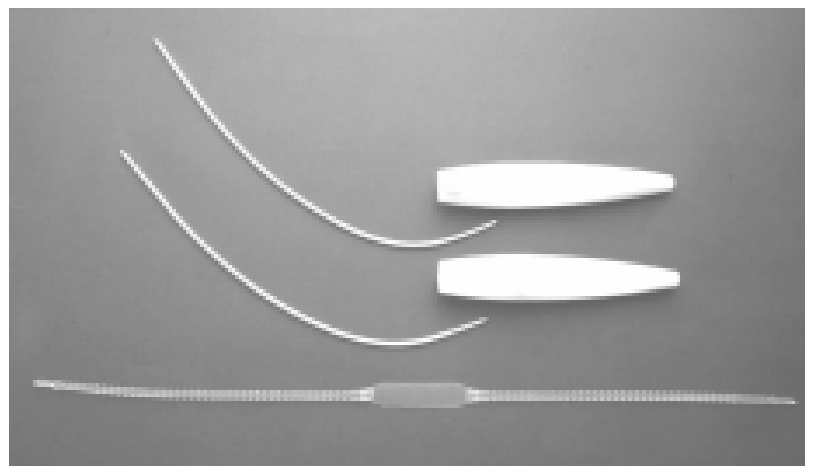

Figura 1. Prueba del cabestrillo SAFYRE; malla de polipropileno y dos columnas de autofijación de polimero de polidimetilsiloxano; aguja versátil que permite realizar un abordaje vaginal o un abordaje suprapúbico. 
incisión vaginal longitudinal de $1,5 \mathrm{~cm}$ de longitud empezando a una distancia de $0,5 \mathrm{~cm}$ del meato de la uretra. Hay que señalar que esta incisión no debe invadir el cuello de la vejiga. La disección se realiza para crear un túnel de $1 \mathrm{~cm}$ lateral a la uretra para introducir en él la aguja de inserción del SAFYRE. Primero se hace avanzar la aguja a través del túnel vaginal hasta que perfore el ligamento inferior de la pelvis a nivel de la parte media de la uretra. Luego se modifica la dirección de la aguja hacia la parte posterior del hueso púbico y se la hace avanzar de forma continua hasta los puntos de referencia situados en el área suprapúbica (abordaje transvaginal). Si el cirujano lo prefiere, también puede utilizarse el abordaje suprapúbico. En este abordaje se hace avanzar la aguja a través de una de las incisiones suprapúbicas, por debajo de la parte posterior del hueso púbico hacia la incisión vaginal. La punta de la aguja sigue en contacto con la parte posterior del hueso púbico hasta que pasa a través de la fascia endopélvica. Utilizando el dedo índice de la otra mano, el cirujano localiza la punta de la aguja y luego la hace avanzar a través de la incisión vaginal. La cistoscopia se realiza para descartar una perforación de la vejiga. Tras quitar la boquilla, el SAFYRE se une a la aguja y se coloca en el área suprapúbica. Se repiten las mismas maniobras en el otro lado. La tensión del cabestrillo se ajusta al nivel adecuado manteniendo unas tijeras de Metzenbaum entre la uretra y el cabestrillo para evitar que haya una tensión excesiva. Se cortan los extremos del cabestrillo y se retiran las tijeras de Metzembaum. No es necesaria más fijación y las incisiones se cierran de la manera habitual. Se deja colocado un catéter interno durante la noche.

\section{Técnica de reajuste}

El procedimiento para tensar el SAFYRE puede realizarse bajo anestesia local o espinal. Como los extremos de las colas de polidimetilsiloxano se pueden palpar fácilmente en el tejido subcutáneo, la anestesia local con una solución de lidocaína al $1 \%$ parece ser el método de elección. Habitualmente, es suficiente con reajustar sólo una cola, sin riesgo de que se produzca una desviación significativa del eje uretral. Se realiza una pequeña incisión sobre el extremo de la cola palpable (cercana a la cara superior del hueso púbico) y se disecciona suavemente estirándola con cuidado hasta conseguir la tensión adecuada (Fig. 2). Durante esta maniobra deben mantenerse unas tijeras de Metzenbaum entre la malla y la uretra para evitar una corrección excesiva. La vejiga se llena con solución salina antes del procedimiento con el fin de comprobar si se produce fuga cuando la paciente tose y al hacer varias veces las maniobras de Valsalva. Generalmente, se propone realizar el reajuste antes de 30 días después de la operación, pero en teoría puede efectuarse en cualquier momento después del procedimiento, debido a que se forma una seudocápsula fibroblástica alrededor de la cola de polidimetilsiloxano del SAFYRE que permite diseccionar y mover fácilmente las colas dentro de esta seudocápsula si es necesario.

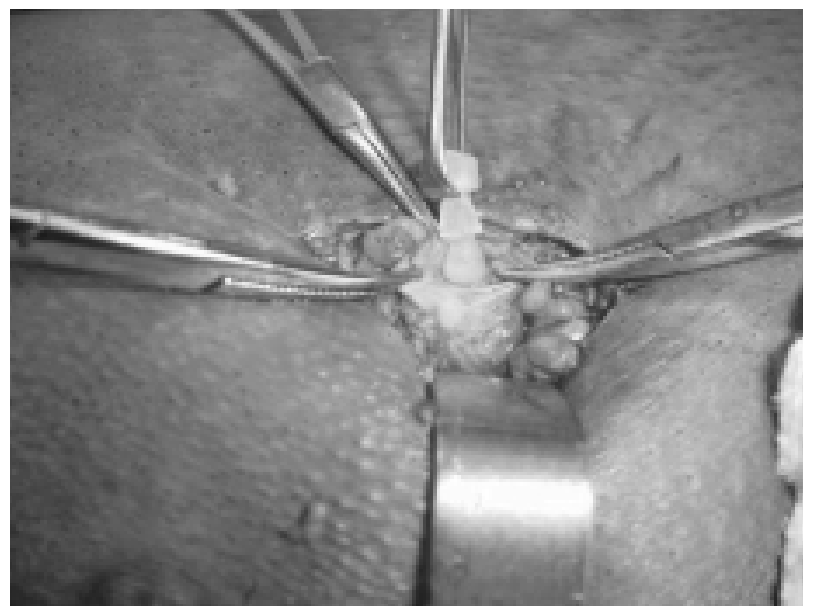

Figura 2. Seudocápusla fibroblástica que rodea la cola de polidimetilsiloxano del SAFYRE. La seudocápusla debe diseccionarse para poder mover la cola y reajustar la cinta.

El procedimiento para aflojar el SAFYRE puede realizarse bajo anestesia espinal, intravenosa o local. Cuando se utiliza la anestesia local, el área suprapúbica (incluida la fascia uretropélvica) y la pared vaginal anterior (incluido el músculo y la fascia rectales) tienen que anestesiarse con una solución de lidocaína al $1 \%$. Se realiza una incisión vaginal longitudinal de $1,5 \mathrm{~cm}$ de longitud empezando a una distancia de $1 \mathrm{~cm}$ del meato de la uretra, y la malla de polipropileno se disecciona de la fascia uretropélvica. Las colas se diseccionan de forma bilateral, agarradas con 
pinzas hemostáticas y estiradas hacia detrás, hasta que se puedan interponer entre la malla y la uretra unas tijeras de Metzenbaum o una pinza en ángulo recto. Se deja colocado un catéter Foley durante la noche.

\section{RESULTADOS}

Características de las pacientes

Las características básicas y los datos demográficos de todas las pacientes se presentan en la Tabla 3.

Tabla 3

Datos demográficos de 126 paciente sometidas al procedimiento de SAFYRE [media (rango) o número \%]

\begin{tabular}{lc}
\hline Variable & $\begin{array}{c}\text { Pacientes } \\
\text { (N = 126) }\end{array}$ \\
\hline Edad (años) & $63(40-71)$ \\
Paridad & $2(0-8)$ \\
$\begin{array}{l}\text { Enfermedad concomitante } \\
\text { (Diabetes, COPD, ETC.) }\end{array}$ & $73(58 \%)$ \\
Operación previa anti-incontinencia & $76(60 \%)$ \\
Situación menopáusica & \\
Premenopáusica & $25(20 \%)$ \\
Menopáusica, con uso de estrógenos & $25(20 \%)$ \\
Menopáusica sin uso de estrógenos & $76(60 \%)$ \\
Síntomas de urgencia pre-operación & $38(30 \%)$ \\
\hline
\end{tabular}

El periodo de seguimiento osciló entre 12 y 30 meses y el periodo de seguimiento medio fue de 14 meses.

\section{Complicaciones del procedimiento}

La duración media del procedimiento fue de 25 minutos y el periodo medio de hospitalización fue de 24 horas (de 12 a 36 horas). Todas las pacientes regresaron a su domicilio el día después de la cirugía.

La tasa global de complicaciones fue del $34 \%$. La mayoría de las mujeres con complicaciones tuvieron más de una complicación.

Se produjeron complicaciones perioperatorias en 3 casos, consistentes en perforación de la pared lateral superior de la vejiga en los 3 casos. En estas pacientes, el catéter Foley se mantuvo durante 48 horas y evolucionaron sin complica- ciones. No hubo casos de sangrado, transfusiones de sangre o perforaciones uretrales o vaginales durante el procedimiento.

El diagnóstico de retención urinaria se estableció cuando el volumen residual, obtenido mediante cateterización uretral tras la micción, era mayor de $100 \mathrm{ml}$. A las pacientes que no podían miccionar de forma espontánea al principio del periodo posoperatorio se les mantuvo en un programa de cateterización intermitente de aclaramiento hasta 4 semanas después de la operación, momento en que se efectúo un procedimiento de aflojamiento si la retención persistía. Todas las pacientes que presentaron micción espontánea al principio del periodo posoperatorio tuvieron un volumen residual tras la micción de menos de $100 \mathrm{ml} \mathrm{y} \mathrm{se} \mathrm{consideró} \mathrm{que} \mathrm{no} \mathrm{tenían}$ retención urinaria. Según estos criterios, se produjo retención urinaria en el posoperatorio en 4 pacientes, las cuales no presentaron micción espontánea 4 semanas después de la operación. En todas estas pacientes se aflojó la tensión del cabestrillo bajo anestesia local y miccionaron espontáneamente, con un alivio completo de los síntomas de irritación y un volumen residual medio tras la micción de $60 \mathrm{ml}$ después del procedimiento. No fue necesario seccionar la cinta en ningún caso.

La principal complicación en el posoperatorio fue sintomas de irritación tras la micción, que se produjeron en 26 pacientes. Estos síntomas ocurrieron al principio del periodo posoperatorio (hasta 4 semanas después de la operación). Hubo 6 casos de infección de la cinta y fue necesario quitar el cabestrillo a 4 pacientes.

En seis pacientes se reajustó más tarde la cinta para tensar el SAFYRE. Estas pacientes presentaron incontinencia urinaria tras la cirugía y el reajuste se realizó para que no presentaran incontinencia. Obtuvimos buenos resultados en cuatro pacientes (curación de las molestias antes de la operación) y mejoría en dos. El reajuste se realizó bajo anestesia local.

\section{Tasas de curación}

Según los criterios de Blaivas y Jacobs ${ }^{9}$, después de un periodo de seguimiento medio de 14 meses, el 92\% de las pacientes presentaron continencia urinaria, el 3\% mejoraron significativa- 
mente y el 5\% no estuvieron satisfechas con el procedimiento y se consideraron fracasos. El cuestionario del impacto (ICQ-SF) mostró una mejora significativa en todas las preguntas en comparación con la evaluación realizada antes de la operación (el 90\% de las pacientes dijeron que tenían menos síntomas urinarios después de la cirugía).

Al final del periodo de seguimiento, la prueba de estrés fue negativa en todas las pacientes con continencia e incontinencia urinarias, y las pacientes del grupo con incontinencia utilizaban, como máximo, 1 unidad diaria de almohadilla perineal.

\section{DISCUSIÓN}

Estudios recientes han confirmado que los cabestrillos pubovaginales y las uretrocistopexias retropúbicas son las técnicas para la incontinencia urinaria por estrés que producen los mejores resultados de continencia después de un seguimiento a largo plazo $^{10}$. Sin embargo, el uso de los cabestrillos implica un periodo considerable de formación quirúrgica y la incómoda necesidad de elegir un lugar de donde obtener la fascia que se va a utilizar en la cirugía, así como riesgos de obstrucción infravesical y otras disfunciones de la vejiga ${ }^{9}$. Por otra parte, las uretrocistopexias retropúbicas implican realizar una incisión abdominal que incrementa la morbilidad y la hospitalización, tienen costes elevados cuando se realizan utilizando un acceso laparoscópico y se aprenden poco a poco ${ }^{5}$. Por tanto, todos los esfuerzos encaminados al desarrollo de técnicas mínimamente invasivas están justificados.

Desde un punto de vista conceptual, el SAFY$\mathrm{RE}$ es un cabestrillo, pero la creación de una zona de soporte suburetral incrementa la resistencia de la uretra y, como consecuencia, no se producen movimientos rotacionales ni descendentes de la uretra cuando la presión abdominal aumenta. Además, facilita la coaptación de la luz de la uretra en reposo y en condiciones de estrés. A diferencia de los cabestrillos pubovaginales clásicos, el SAFYRE se aplica en el tercio medial de la uretra donde se insertan los ligamentos pubouretrales responsables de la estabilidad natural de la uretra $^{11}$. El mantenimiento del cabestrillo en una posición adecuada se consigue gracias a sus estructuras salientes y entrantes, que crean un efecto tipo gancho en las fascias de la pelvis y en el músculo liso abdominal, así como reacciones inflamatorias locales.

La inserción del SAFYRE se realiza sin tensión y no está restringida por el tamaño del cuello de la vejiga como ocurre con los cabestrillos convencionales. Aunque en nuestro estudio la tasa de retención urinaria fue del $3,1 \%$, el SAFYRE permitió realizar un reajuste de la tensión en el posoperatorio sin dificultades. A estas pacientes se les reajustó el cabestrillo bajo anestesia local y miccionaron espontáneamente, presentando un alivio completo de los síntomas de irritación y un volumen residual medio después de la micción de $60 \mathrm{ml}$.

Nuestros resultados confirman la viabilidad y la seguridad del SAFYRE para el tratamiento de la incontinencia urinaria. Desde el primer informe publicado por Ulmsten ${ }^{12}$, el TVT se ha convertido en un método popular para la incontinencia urinaria por estrés genuina. Una revisión reciente de 11 artículos en los que se utilizaron criterios de valoración objetivos mostró una tasa de curación del 87,3\% tras una media de 17 meses después de la cirugía ${ }^{13}$. El uso del abordaje suprapúbico y transvaginal se ha asociado a varias complicaciones, tales como perforación de la vejiga y lesiones del obturador, de los vasos iliaco externo, femoral y epigástrico inferior, de los nervios y del intestino delgado ${ }^{14,15}$. Tras realizar 140 procedimientos SPARC (en el arco suprapúbico) con cabestrillo, se determinó el hematócrito en el día 1 del posoperatorio en las últimas 57 pacientes independientemente de la pérdida de sangre en el quirófano. Un total de 6 pacientes necesitaron intervenciones al principio del periodo posoperatorio, entre ellas transfusiones en 4 pacientes inmediatamente después de la operación por sangrado retropúbico. Una paciente presentó drenaje de una incisión suprapúbica el día 4 del posoperatorio. Tenía una perforación a través de un asa del intestino delgado que requirió la resección de un segmento corto del intestino y la retirada del cabestrillo ${ }^{16}$.

Aunque en este estudio no se comparó el SAFYRE con otras técnicas mínimamente invasivas, como la TVT o similares, existen diferencias específicas y significativas entre las propiedades 
bioquímicas y biomecánicas del SAFYRE y las de las otras técnicas. A diferencia de la TVT o de otros cabestrillos de polipropileno mínimamente invasivos, la superficie lisa de la malla del SAFY$\mathrm{RE}$ permite realizar fácilmente un ajuste primario durante el implante e incluso durante el reajuste eventual, además de mantener su resistencia y su forma debido a su baja tasa de deformidad. Asimismo, la elasticidad de las colas de polidimetilsiloxano permite realizar movimientos precisos en función de los cambios en la presión abdominal del paciente y de esta forma el dispositivo actúa como un soporte dinámico. Por otra parte, el sistema de autofijación del SAFYRE es único en lo referente a la capacidad de reajuste en el posoperatorio. El procedimiento es mínimamente invasivo y no es necesario hacer una incisión abdominal grande para obtener la fascia ni para fijar el cabestrillo a la aponeurosis del músculo recto abdominal como ocurre con los cabestrillos clásicos. Su capacidad de reajuste permite hacer ajustes tardíos de la tensión del cabestrillo en las pacientes que presentan incontinencia o retención urinaria persistentes, evitando así la necesidad de intervenciones de cirugía mayor como uretrolisis o de insertar otro cabestrillo y reduciendo los costes.

La coherencia con los principios fisiológicos de la incontinencia urinaria femenina, las tasas de curación de más del $90 \%$ y los efectos beneficiosos indiscutibles de los reajustes de la tensión en el posoperatorio indican que este procedimiento constituye un paso adelante prometedor en el tratamiento quirúrgico de la IUE.

\section{REFERENCIAS}

1. Goebel R. Zur operativen Beseitigung der Angeborenen Incontinentia Vesicae. Z Gynak Urol 1910;2:187.

2. McGuire EJ, Fitzpatrick CC, Wan J, Bloom D, Sanvordenker $\mathrm{J}$, Ritchey M et al. Clinical assessment of Urethral Sphincter Function. J Urol 1993;150:1452.

3. Ulmsten U, Henriksson L, Johnson P, Varhos G: An ambulatory surgical procedure under local anesthesia for treatment of female urinary incontinence. Int Urogynecol J 1996;7:81.
4. Palma PCR, Riccetto CLZ, Dambros M, Thiel M, Fraga R, Herrmann V, Netto NR Jr. Pubovaginal Safyre: A new readjustable minimally invasive sling for female urinary stress incontinence. The iberoamerican Safyre study group. Urología Panamericana 2002;14(4):22.

5. Petros P, Ulmsten U: An integral theory and its method for the diagnosis and management of female urinary incontinence. Scand J Urol Nephrol 1993;153:1.

6. Avery K, Donovan J, Abrams, P. Validation of a new questionnaire for incontinence: The International Consultation on Incontinence Questionnaire (ICIQ). Neurourol and Urodynamics 2001;4:510.

7. Schrepferman CG, Griebling TL, Nygaard IE, Kreder KJ: Resolution of urge symptoms following sling cystourethropexy. J Urol 2000;164:1628.

8. Kaplan SA, Te AE, Young GP: Prospective analysis of 373 consecutive women with stress urinary incontinence treated with a vaginal wall sling the Columbia-Cornell University experience. J Urol 2000;164:1623.

9. Blaivas JG, Jacobs BZ. Pubovaginal Fascial Sling for the treatment of complicated stress urinary incontinence. J Urol 1991;145:1214.

10. Norris JP, Breslin DS, Staskin DR: Use of synthetic material in sling surgery: a minimally invasive approach. $\mathrm{J}$ Endourol 1996;10:227.

11. Ulmstem U, Falconer C, Johnson P, Jomaa M, Lannér L, NilssonC G, Olsson I: A multicenter study of tension-free vaginal tape (TVT) for surgical treatment of stress urinary incontinence. Int Urogynecol J 1998;9:210.

12. Ulmsten U, Henriksson L, Johnson P, Varhos G. An ambulatory surgical procedure under local anesthesia for treatment o of female urinary incontinence. Int Urogynecol $\mathrm{J}$ Pelvic Floor Dysfunction 1996;7:81.

13. Perchers UM, Tunn R, Buczkowski M, Perucchini D. Tension-free vaginal tape for the treatment of Stress Urinary Incontinence. Clin Obstet Gynecol 2000;43:670.

14. Neuman M. Infected hematoma following tension-free vaginal tape implantation. J Urol 2002;168:2549.

15. Kuuva N, Nilsson CG. A nationwide analysis of complications associated with the tension-free vaginal tape (TVT) procedure. Acta Obstet Gynecol Scand 2002;81:72.

16. Kobashi KC, Govier FE: Perioperative complications: the first 140 polypropylene pubovaginal slings. J Urol 2003; 170(5): 1918.

Dra. Miriam Dambros

Rua Tiradentes, 426 - apto. 51

Vial Itapura

13023-190 Campinas

SP, Brasil

(Trabajo recibido el 27 julio de 2004) 